\title{
Fixed Point Approximations with Finite Relatively Nonexpansive Maps in Some Real Banach Spaces
}

\author{
Umar Yusuf Batsari \\ Department of Mathematics and Statistics, Hassan Usman Katsina Polytechnic, Katsina State, Nigeria. \\ Email: uyub2k@yahoo.com
}

\begin{abstract}
Let $E$ be a uniformly smooth and uniformly convex real Banach space and $C$ be a nonempty, closed and convex subset of $E$. In this paper, it is shown that $\left\{x_{n}\right\}$ obtained from Batsari's[21] CQ algorithm with relatively nonexpansive maps converges strongly to a point $\widehat{x}$ which is also a common fixed point of some finite relatively nonexpansive mappings and solves a system of equilibrium problems in $E$. The result obtained improves some existing results in the literature.
\end{abstract}

Keywords: relatively nonexpansive mappings; equilibrium problems; uniformly smooth space; uniformly convex space; bounded sequence.

\section{Introduction}

Let $E$ be a real Banach space and $E^{*}$ be its dual Space. Let $C$ be a nonempty, closed and convex subset of $E$. Let $\left\{f_{k}\right\}$ for $k \in \Gamma$ be a family of bifunctions from $C \times C$ to $\mathbb{R}$, where $\mathbb{R}$ is the set of real numbers and $\Gamma$ is an arbitrary index set. The equilibrium problems are to find $\hat{x} \in C$ such that,

$$
f_{k}(\hat{x}, y) \geq 0, \forall y \in C \text { and } k \in \Gamma \text {. }
$$

If $\Gamma$ is a singletone then, problem (1) becomes the following equilibrium problem of finding $\hat{x} \in C$ such that,

$$
f(\hat{x}, y) \geq 0, \forall y \in C .
$$

The solution set of (1) and (2) are denoted by $E P\left(f_{k}\right)$ and $E P(f)$ respectively.

Note: For solving the equilibrium problems (1) we assume that, $\forall k \in \Gamma$ the bi-function $f_{k}$ satisfies the following conditions:

(A1) $f_{k}(x, x)=0, \quad \forall x \in C$.

(A2) $f_{k}$ is monotone, i.e $f_{k}(x, y)+f_{k}(y, x) \leq 0$, for any $x, y \in C$.

(A3) For each $x, y, z \in C$,

$$
\limsup _{t \rightarrow 0} f_{k}(t z+(1-t) x, y) \leq f_{k}(x, y) .
$$

(A4) $f_{k}(x, \cdot)$ is convex and lower semicontinous for each $x \in C$.

Let $X, Y$ be sets such that $X \subset Y$, consider a map $S: X \rightarrow Y$, a set $F(S)=\{z \in X: S z=z\}$ is called the fixed point set of $S$.

A mapping $S: C \longrightarrow E$ is called nonexpansive if

$$
\|S x-S y\| \leq\|x-y\|, \quad \forall x, y \in C .
$$

Let $E$ be a real Banach space and $E^{*}$ be its dual, for all $x \in E$ and $x^{*} \in E^{*}$ we denote the value of $x^{*}$ at $x$ by $\left\langle x, x^{*}\right\rangle$. So, the duality mapping $J$ on $E$ is defined by

$$
J(x)=\left\{x^{*} \in E^{*}:\left\langle x, x^{*}\right\rangle=\|x\|^{2}=\left\|x^{*}\right\|^{2}\right\}, \quad \forall x \in E .
$$

A Banach space $E$ is said to be strictly convex if $\frac{\|x+y\|}{2}<1 \forall x, y \in E$ with $\|x\|=\|y\|=1$ and $x \neq y$.

A Banach space $E$ is said to be uniformly convex if for each $\varepsilon \in(0,2]$ there exists $\delta>0$ such that 
$\frac{\|x+y\|}{2} \leq 1-\delta, \forall x, y \in \mathrm{E}$ with $\|x\|=\|y\|=1$ and $\|x-y\| \geq \varepsilon$.

A Banach space $E$ is said to be smooth if the limit

$$
\lim _{t \rightarrow 0} \frac{\|x+t y\|-\|x\|}{t}
$$

exists $\forall x, y \in S(E)$ where $S(E)=\{z \in E:\|z\|=1\}$. Also, the space $E$ is said to be uniformly smooth if the above limit exists uniformly for $x, y \in S(E)$.

The following definition can be found in [11]. Let $E$ be a smooth, strictly convex and reflexive Banach space, let $C$ be a nonempty, closed and convex subset of $E$, define a function $\phi: E \times E \rightarrow \mathbb{R}$ by

$$
\phi(x, y)=\|x\|^{2}-2\langle x, J y\rangle+\|y\|^{2}, \quad \forall x, y \in E .
$$

It is obvious from the definition of $\phi(x, y)$ that,

$$
(\|x\|-\|y\|)^{2} \leq \phi(x, y) \leq(\|x\|+\|y\|)^{2}, \quad \forall x, y \in C .
$$

Let $E$ be a smooth, strictly convex and reflexive Banach space, $C$ be a nonempty, closed and convex subset of $E, S: C \rightarrow C$ be a mapping and $F(S)$ be the set of fixed points of $S$. A point $p \in C$ is said to be an asymptotic fixed point of $S[15]$ if there exists a sequence $\left\{x_{n}\right\} \subset C$ such that $x_{n} \rightarrow p$ and $\left\|x_{n}-S x_{n}\right\| \rightarrow 0$. The set of all asymptotic fixed points of $S$ is denoted by $\widetilde{F}(S)$.

A mapping $S: C \rightarrow C$ is said to be relatively nonexpansive[15] if

1. $F(S) \neq \emptyset$.

2. $F(S)=\widetilde{F}(S)$.

3. $\phi(p, S y) \leq \phi(p, y), \quad \forall y \in C, p \in F(S)$.

From Alber[17], the generalized projection $\Pi_{C}$ from $E$ onto $C$ is defined by

$$
\Pi_{C}(x)=\arg \min _{y \in C} \phi(y, x), \quad \forall x \in E .
$$

A Banach space $E$ is said to have a Kadec-Klee property[15] if for every sequence $\left\{x_{n}\right\}$ in $E, x_{n} \rightarrow$ $x$ and $\left\|x_{n}\right\| \rightarrow\|x\| \Longrightarrow\left\|x_{n}-x\right\| \rightarrow 0$ as $n \rightarrow \infty$.

Remark : The following basic property is true: We can find in Cioranescu [7] that, if $E$ is uniformly smooth Banach space, then $J$ is uniformly continuous on each bounded subset of $E$.

The problem (1) is very general in the sense that, it includes as special cases: optimization problems, variational inequalities, mini-max problems, Nash equilibrium problem in noncooperative games and others; see for example [4,11]. Various methods have been proposed to solve problem (1).

In 1953 Mann[18] introduced a well known classical iterative process to approximate a fixed point of a nonexpansive mapping. The iterative process is defined as

$$
x_{n+1}=\alpha_{n} x_{n}+\left(1-\alpha_{n}\right) T\left(x_{n}\right) \quad n \geq 0,
$$

where initial element $x_{0}$ is taken in $C$ arbitrarily and the sequence $\left\{\alpha_{n}\right\}_{n=0}^{\infty}$ in $[0,1]$ satisfies

1. $\lim _{n \rightarrow \infty} \alpha_{n}=0$.

2. $\sum_{n=1}^{\infty} \alpha_{n}=\infty$.

But, Mann's iterative process has only weak convergence, even in a Hilbert space. Therefore, many authors try to modify Mann's iterative process in order to have strong convergence, the so called hybrid projection iterative method is one of such modifications.

The hybrid projection iterative algorithm(HPIA) was introduced initially by Haugazeau[19] in 1968 . For over 40 years, HPIA has received rapid developments.

In 2006, Nakajo et al.[19] proposed the following modification of Mann's iterative scheme for a nonexpansive mapping $T$ in a real Hilbert space $H$ :

$$
\left\{\begin{array}{l}
x_{0} \in C \text { chosen arbitrarily, } \\
y_{n}=\alpha_{n} x_{n}+\left(1-\alpha_{n}\right) T x_{n}, \\
C_{n}=\left\{z \in C:\left\|y_{n}-z\right\| \leq\left\|x_{n}-z\right\|\right\} \\
Q_{n}=\left\{z \in C:\left\langle x_{n}-z, x_{0}-x_{n}\right\rangle \geq 0\right\} \\
x_{n+1}=P_{C_{n} \cap Q_{n}}\left(x_{0}\right),
\end{array}\right.
$$


where $C$ is a closed, convex subset of $H, P_{C_{n} \cap Q_{n}}$ denotes the metric projection from $H$ onto a nonempty, closed and convex subset $C_{n} \cap Q_{n}$ of $H$. They proved that, if the sequence $\left\{\alpha_{n}\right\}$ is bounded above by one then, the sequence $\left\{x_{n}\right\}$ generated by (5) converges strongly to $P_{F(T)}\left(x_{0}\right)$, where, $F(T)$ is the fixed point set of $T$.

In 2009, Takahashi and Zembayashi[15] proposed the following modificaion of Mann's iterative scheme for relatively nonexpansive mapping $S$, in a real uniformly convex and uniformly smooth Banach space $E$ :

$$
\left\{\begin{array}{l}
x_{0}=x \in C, \\
y_{n}=J^{-1}\left(\alpha_{n} J x_{n}+\left(1-\alpha_{n}\right) J S x_{n}\right), \\
u_{n} \in C \text { such that } f\left(u_{n}, y\right)+\frac{1}{r_{n}}\left\langle y-u_{n}, J u_{n}-J y_{n}\right\rangle \geq 0, \quad \forall y \in C, \\
H_{n}=\left\{z \in C: \phi\left(z, u_{n}\right) \leq \phi\left(z, x_{n}\right)\right\} \\
W_{n}=\left\{z \in C:\left\langle x_{n}-z, J x-J x_{n}\right\rangle \geq 0\right\} \\
x_{n+1}=\Pi_{H_{n} \cap W_{n}} x,
\end{array}\right.
$$

where $J$ is the duality mapping on $E$ and $f$ is a bifunction from $C \times C$ to $\mathbb{R}$ satisfying conditions (A1)-(A4). They proved that, if $\liminf _{n \rightarrow \infty}\left(1-\alpha_{n}\right)>0$ and $\left\{r_{n}\right\} \subset[a, \infty)$ for some $a>0$ then, the sequence $\left\{x_{n}\right\}$ generated by (6) converges strongly to $\Pi_{F(S) \cap E P(f)} x$, where $\Pi_{F(S) \cap E P(f)}$ is the generalized projection of $E$ onto $F(S) \cap E P(f)$.

Duan and Zhao[8] studied a new hybrid methods for equilibrium problems and strictly pseudocontractions.

In 2010 P. Duan[9] proposed the following iterative scheme for some finite family of strictly pseudocontraction mappings and systems of equilibrium problems in a real Hilbert space $H$ :

$$
\left\{\begin{array}{l}
u_{n}=T_{r_{M, n}}^{f_{M}} T_{r_{M-1, n}}^{f_{M-1}} \ldots T_{r_{2, n}}^{f_{2}} T_{r_{1, n}}^{f_{1}} x_{n} \\
A_{n}^{\lambda_{n}}=\lambda_{n} \mathrm{I}+\left(1-\lambda_{n}\right) A_{n} \\
y_{n}=\alpha_{n} x_{n}+\left(1-\alpha_{n}\right) A_{n}^{\lambda_{n}} u_{n} \\
C_{n}=\left\{z \in C:\left\|y_{n}-z\right\| \leq\left\|x_{n}-z\right\|\right\} \\
Q_{n}=\left\{z \in C:\left\langle x_{n}-z, x_{1}-x_{n}\right\rangle \geq 0\right\} \\
x_{n+1}=\mathrm{P}_{C_{n} \cap Q_{n}} x_{1}
\end{array}\right.
$$

where $\left\{\alpha_{n}\right\} \subset[0, a],\left\{\lambda_{n}\right\} \subset[l, b]$ for some $a, l \in[0,1), b \in[l, 1)$ and $\left\{r_{k, n}\right\} \subset(0, \infty)$ satisfying $\liminf _{n \rightarrow \infty} r_{k, n}>0$ for all $k \in\{1,2, \ldots, M\}$. Then, she proved that, the sequence $\left\{x_{n}\right\}$ generated by $(7)$ converges strongly to $P_{\Omega} x_{1}$, where $\Omega=\cap_{i=1}^{N} F\left(\mathrm{~S}_{i}\right) \cap\left(\cap_{K=1}^{M} \mathrm{EP}\left(\mathrm{f}_{k}\right)\right)$. She also proved a strong convergence using cyclic algorithm in the same journal article.

Recently in 2014, U.Y. Batsari[21] proposed the following iterative scheme for some finite family of $\phi$-nonexpansive mappings and systems of equilibrium problems in a real Banach space $E$ :

$$
\left\{\begin{array}{l}
y_{n}=j^{-1}\left(\alpha_{n} j x_{n}+\left(1-\alpha_{n}\right) j A_{N}^{\lambda_{n}} x_{n}\right) \\
u_{n}=T_{r_{M, n}}^{f_{M}} T_{r_{M-1, n}}^{f_{M-1}} \ldots T_{r_{2}, n}^{f_{2}} T_{r_{1, n}}^{f_{1}} y_{n} \\
C_{n}=\left\{z \in C: \phi\left(z, u_{n}\right) \leq \phi\left(z, x_{n}\right)\right\} \\
Q_{n}=\left\{z \in C:\left\langle x_{n}-z, j x_{1}-j x_{n}\right\rangle \geq 0\right\} \\
x_{n+1}=\Pi_{C_{n} \cap Q_{n}} x_{1}
\end{array}\right.
$$

for every $n \in \mathbb{N}$, where $j \in J$ and $J$ is the duality mapping on $E,\left\{\alpha_{n}\right\} \subset[0, a]$ such that $\lim \inf _{n \rightarrow \infty} \alpha_{n}(1-$ $\left.\alpha_{n}\right)>0$ for some $a \in[0,1),\left\{\lambda_{n}\right\} \subset[0,1)$, and $\left\{r_{k}, n\right\} \subset(0, \infty)$ satisfying $\liminf _{n \rightarrow \infty} r_{k, n}>0$ for all $\mathrm{k}$ $\in\{1,2,3, \cdots, M\}$ then, $\left\{x_{n}\right\}$ generated by (8) converges strongly to $\Pi_{\Omega} x_{1}$.

In this paper, motivated by [15] and [21], a strong convergence theorem for approximating common fixed points for finite family of relatively nonexpansive maps and common solution of finite family of equilibrium problems in a uniformly convex and uniformly smooth real Banach space $E$ is studied; which is an improvement to [15] that consider only one relatively nonexpansive map and one equilibrium problem. Also, the importance of relatively nonexpansive maps especially the resolvent of maximal monotone operators inspires the researcher to substitute $\phi$-nonexpansive maps with relatively nonexpansive maps as used in [21]. 


\section{Preliminaries}

We shall make use of the following results.

Lemma 2.1 [17]. Let $C$ be a nonempty, closed and convex subset of a smooth, strictly convex and reflexive Banach space $E$ then,

$$
\phi\left(x, \Pi_{C} y\right)+\phi\left(\Pi_{C} y, y\right) \leq \phi(x, y), \quad \forall x \in C \text { and } y \in E .
$$

Lemma 2.2 [11]. Let $E$ be a smooth and uniformly convex Banach space, let $\left\{x_{n}\right\}$ and $\left\{y_{n}\right\}$ be sequences in E such that either $\left\{x_{n}\right\}$ or $\left\{y_{n}\right\}$ is bounded, if $\lim _{n \rightarrow \infty} \phi\left(x_{n}, y_{n}\right)=0$ then, $\lim _{n \rightarrow \infty}\left\|x_{n}-y_{n}\right\|=0$.

Lemma 2.3 [6]. Let $E$ be a uniformly convex Banach space and $r>0$ then, there exists a strictly increasing, continuous and convex function $g:[0,2 r] \rightarrow \mathbb{R}$ such that, $g(0)=0$ and

$$
\|t x+(1-t) y\|^{2} \leq t\|x\|^{2}+(1-t)\|y\|^{2}-t(1-t) g(\|x-y\|),
$$

for all $\mathrm{x}, \mathrm{y} \in B_{r}$ and $t \in[0,1]$, where $B_{r}=\{z \in E:\|z\| \leq r\}$.

Lemma 2.4 [15]. Let $C$ be a nonempty, closed and convex subset of a uniformly smooth, strictly convex and reflexive Banach space $E$. Let $f$ be a bifunction from $C \times C$ to $\mathbb{R}$ satisfying (A1)-(A4). For $r>0$ define a function $T_{r}: E \rightarrow C$ by

$$
T_{r}(x)=\left\{z \in C: f(z, y)+\frac{1}{r}\langle y-z, J z-J x\rangle \geq 0, \quad \forall y \in C\right\},
$$

for all $x \in E$ then, the following holds

1. $T_{r}$ is single valued.

2. $T_{r}$ is firmly nonexpansive type, i.e for all $x, y \in E$

$$
\left\langle T_{r} x-T_{r} y, J T_{r} x-J T_{r} y\right\rangle \leq\left\langle T_{r} x-T_{r} y, J x-J y\right\rangle .
$$

3. $F\left(T_{r}\right)=E P(f)$.

4. $E P(f)$ is closed and convex.

5. $T_{r}$ is relatively nonexpansive.

Now, in view of the above lemma define the maps $T_{r_{k, n}}^{f_{k}}: E \rightarrow C$ for $k \in\{1,2, \cdots M\}$ by $T_{r_{k, n}}^{f_{k}}(x)=$ $\left\{z \in C: f_{k}(z, y)+\frac{1}{r_{k, n}}\langle y-z, J z-J x\rangle \geq 0, \forall y \in C\right\} \forall x \in E, n \in \mathbb{N}$ and $\left\{r_{k, n}\right\} \subseteq(0, \infty)$. Let $\Theta_{n}^{k}=T_{r_{k, n}}^{f_{k}} T_{r_{k-1, n}}^{f_{k-1}} \ldots T_{r_{2}, n}^{f_{2}} T_{r_{1, n}}^{f_{1}}$ then,

1. If $\mathrm{h} \in \bigcap_{k=1}^{M} \operatorname{EP}\left(f_{k}\right)$ we have $\Theta_{n}^{k} h=h, \forall k \in\{1,2, \cdots M\}$.

2. $\Theta_{n}^{M}$ is relatively nonexpansive hence, $\phi\left(h, \Theta_{n}^{M} x\right) \leq \phi(h, x), \quad \forall h \in F\left(\Theta_{n}^{M}\right)$ and $x \in C$.

Lemma 2.5 [15]. Let $C$ be a nonempty, closed and convex subset of a smooth, strictly convex and reflexive Banach space $E$, for $x \in \mathrm{E}$ and $q \in F\left(T_{r}\right)$ we have

$$
\phi\left(q, T_{r} x\right)+\phi\left(T_{r} x, x\right) \leq \phi(q, x) .
$$

Lemma 2.6 [17]. Let $C$ be a nonempty, closed and convex subset of a smooth, strictly convex and reflexive Banach space $E$, let $x \in E$ and $z \in C$ then,

$$
z=\Pi_{C} x \Longleftrightarrow\langle y-z, J x-J z\rangle \leq 0, \forall y \in C .
$$

Also, for $x, y \in E$,

$$
\phi(x, y)=0 \Longleftrightarrow x=y
$$

Lemma 2.7. Let $E$ be a real Banach Space. Given a Natural number $N$, assume for each $1 \leq i \leq N$, $S_{i}$ is a well defined mapping from a subset of $E$ to $E$. Let $A_{n}=\sum_{i=1}^{N} \alpha_{i}^{(n)} S_{i}$ and $A_{n}^{\lambda_{n}}=\lambda_{n} I+\left(1-\lambda_{n}\right) A_{n}$, where $\left\{\alpha_{i}^{(n)}\right\}_{i=1}^{N}$ is a sequence of positive real numbers such that $\sum_{i=1}^{N} \alpha_{i}^{(n)}=1 \forall n \in \mathbb{N}$, suppose $\left\{S_{i}\right\}_{i=1}^{N}$ has a common fixed point $\forall N \in \mathbb{N}$ then, $\bigcap_{i=1}^{N} F\left(S_{i}\right)=F\left(A_{n}\right)=F\left(A_{n}^{\lambda_{n}}\right), \quad \forall \lambda_{n} \in(0,1)$. 
Proof. To prove the lemma, it is enough to show that the result holds for $N=2$. Now, let $N=2$,

First Claim: $\bigcap_{i=1}^{2} F\left(S_{i}\right) \subseteq F\left(A_{n}\right)$.

Let $x \in \bigcap_{i=1}^{2} F\left(S_{i}\right)$ then $x=S_{i} x$ for $i=1,2$. So,

$$
\begin{aligned}
A_{n} x & =\left(\alpha_{1}^{(n)} S_{1}+\alpha_{2}^{(n)} S_{2}\right) x \\
& =\alpha_{1}^{(n)} S_{1} x+\alpha_{2}^{(n)} S_{2} x \\
& =\alpha_{1}^{(n)} x+\alpha_{2}^{(n)} x \\
& =\left(\alpha_{1}^{(n)}+\alpha_{2}^{(n)}\right) x \\
& =x .
\end{aligned}
$$

Thus, $\bigcap_{i=1}^{2} F\left(S_{i}\right) \subseteq F\left(A_{n}\right)$.

Conversely, let $x \in F\left(A_{n}\right)$ then,

$$
\begin{aligned}
0 & =\left\|x-A_{n} x\right\| \\
& =\left\|x-\left(\alpha_{1}^{(n)} S_{1} x+\alpha_{2}^{(n)} S_{2} x\right)\right\| \\
& =\left\|\left(\alpha_{1}^{(n)}+\alpha_{2}^{(n)}\right) x-\left(\alpha_{1}^{(n)} S_{1} x+\alpha_{2}^{(n)} S_{2} x\right)\right\| \\
& =\left\|\left(\alpha_{1}^{(n)} x-\alpha_{1}^{(n)} S_{1} x\right)-\left(\alpha_{2}^{(n)} S_{2} x-\alpha_{2}^{(n)} x\right)\right\| \\
& \geq\left\|\alpha_{1}^{(n)} x-\alpha_{1}^{(n)} S_{1} x\right\|-\left\|\alpha_{2}^{(n)} S_{2} x-\alpha_{2}^{(n)} x\right\| \mid,
\end{aligned}
$$

this implies that,

$$
0=\left|\alpha_{1}^{(n)}\left\|x-S_{1} x\right\|-\alpha_{2}^{(n)}\left\|S_{2} x-x\right\|\right|
$$

which means that,

$$
0=\alpha_{1}^{(n)}\left\|x-S_{1} x\right\|-\alpha_{2}^{(n)}\left\|S_{2} x-x\right\|, \quad \forall n \in \mathbb{N},
$$

from which we have

$$
\alpha_{1}^{(n)}\left\|x-S_{1} x\right\|=\alpha_{2}^{(n)}\left\|x-S_{2} x\right\|, \quad \forall n \in \mathbb{N} .
$$

That is $x=S_{1} x$ and $x=S_{2} x$. So, $x \in \bigcap_{i=1}^{2} F\left(S_{i}\right)$ and thus, $F\left(A_{n}\right) \subseteq \bigcap_{i=1}^{2} F\left(S_{i}\right)$. Therefore, $\bigcap_{i=1}^{2} F\left(S_{i}\right)=F\left(A_{n}\right)$.

Second Claim: $F\left(A_{n}\right)=F\left(A_{n}^{\lambda_{n}}\right)$.

Let $x \in F\left(A_{n}\right)$ then,

$$
\begin{aligned}
A_{n}^{\lambda_{n}} x & =\lambda_{n} x+\left(1-\lambda_{n}\right) A_{n} x \\
& =\left(\lambda_{n}+\left(1-\lambda_{n}\right)\right) x \\
& =x
\end{aligned}
$$

which means, $F\left(A_{n}\right) \subseteq F\left(A_{n}^{\lambda_{n}}\right)$.

Conversely, let $x \in F\left(A_{n}^{\lambda_{n}}\right)$,

$$
\begin{aligned}
0 & =\left\|x-A_{n}^{\lambda_{n}} x\right\| \\
& =\left\|x-\left(\lambda_{n} x+\left(1-\lambda_{n}\right) A_{n} x\right)\right\| \\
& =\left\|\left(1-\lambda_{n}\right) x-\left(1-\lambda_{n}\right) A_{n} x\right\| \\
& =\left(1-\lambda_{n}\right)\left\|x-A_{n} x\right\| .
\end{aligned}
$$

Therefore, $A_{n} x=x$ and so, $F\left(A_{n}^{\lambda_{n}}\right) \subseteq F\left(A_{n}\right)$. Hence, $F\left(A_{n}\right)=F\left(A_{n}^{\lambda_{n}}\right)$. 


\section{Main Result}

Let $E$ be a uniformly smooth and uniformly convex real Banach space. Let $C$ be a nonempty, closed and convex subset of $E$. Let $f_{k}$ for $k \in\{1,2,3, . . M\}$, be a bifunction from $C \times C$ to $\mathbb{R}$ which satisfies conditions (A1)-(A4) and $f_{k}(x, y) \leq f_{k+1}(x, y) \quad \forall x, y \in C$. Let $V=\left\{\alpha_{i} S_{i}: C \rightarrow C \mid i=1,2, \cdots\right.$ Nand $\alpha_{i} \in$ $(0,1]\}$ be a convex set of relatively nonexpansive maps and contain identity map I. Assume that, $\Omega=\cap_{i=1}^{N} F\left(S_{i}\right) \cap\left(\cap_{k=1}^{M} E P\left(f_{k}\right)\right) \neq \emptyset$. Assume also that $\left\{\eta_{i}^{(n)}\right\}_{i=1}^{N}$ is a finite sequence of positive numbers such that $\Sigma_{i=1}^{N} \eta_{i}^{(n)}=1$ for all $n \in \mathbb{N}$ and $\inf _{n \geq 1} \eta_{i}^{(n)}>0$ for all $i, 1 \leq i \leq N$. Let the mapping $A_{N}^{\lambda_{n}}$ be defined by

$$
A_{N}^{\lambda_{n}}=\lambda_{n} I+\left(1-\lambda_{n}\right) \sum_{i=1}^{N} \eta_{i}^{(n)} S_{i} .
$$

Given $x_{1} \in C$, let $\left\{x_{n}\right\},\left\{u_{n}\right\}$ and $\left\{y_{n}\right\}$ be sequences which are generated by the following algorithm:

$$
\left\{\begin{array}{l}
y_{n}=j^{-1}\left(\alpha_{n} j x_{n}+\left(1-\alpha_{n}\right) j A_{N}^{\lambda_{n}} x_{n}\right), \\
u_{n}=T_{r_{M, n}}^{f_{M}} T_{r_{M-1, n}}^{f_{M-1}} \ldots T_{r_{2}, n}^{f_{2}} T_{r_{1, n}}^{f_{1}} y_{n} \\
C_{n}=\left\{z \in C: \phi\left(z, u_{n}\right) \leq \phi\left(z, x_{n}\right)\right\} \\
Q_{n}=\left\{z \in C:\left\langle x_{n}-z, j x_{1}-j x_{n}\right\rangle \geq 0\right\} \\
x_{n+1}=\Pi_{C_{n} \cap Q_{n}} x_{1},
\end{array}\right.
$$

for every $n \in \mathbb{N}$, where $j \in J$ and $J$ is the duality mapping on $E,\left\{\alpha_{n}\right\} \subset[0, a]$ such that $\liminf _{n \rightarrow \infty} \alpha_{n}(1-$ $\left.\alpha_{n}\right)>0$ for some $a \in[0,1),\left\{\lambda_{n}\right\} \subset[0,1)$, and $\left\{r_{k}, n\right\} \subset(0, \infty)$ satisfying $\liminf _{n \rightarrow \infty} r_{k, n}>0$ for all $\mathrm{k}$ $\in\{1,2,3, \cdots, M\}$ then, $\left\{x_{n}\right\}$ converges strongly to $\Pi_{\Omega} x_{1}$.

Proof. Let us denote $\Theta_{n}^{k}=T_{r_{k, n}}^{f_{k}} T_{r_{k-1, n}}^{f_{k-1}} \cdots T_{r_{2, n}}^{f_{2}} T_{r_{1, n}}^{f_{1}}$ for all $n \in \mathbb{N}$ and $k \in\{1,2,3, \cdots M\}$, then $u_{n}=$ $\Theta_{n}^{M} y_{n}$.

We start by showing that $\left\{x_{n}\right\}$ is well defined.

To prove this, it is enough to show $C_{n} \cap Q_{n}$ is nonempty, closed and convex subset of $E \forall n \in \mathbb{N}$. Clearly $C_{n}, Q_{n}$ are closed and $Q_{n}$ is convex. Also,

$$
\phi\left(z, u_{n}\right) \leq \phi\left(z, x_{n}\right) \Longleftrightarrow\left\|u_{n}\right\|^{2}-\left\|x_{n}\right\|^{2}-2\left\langle z, j u_{n}-j x_{n}\right\rangle \leq 0 .
$$

This implies that, $C_{n}$ is convex. So, $C_{n} \cap Q_{n}$ is closed and convex.

Next, we show that $C_{n} \cap Q_{n} \neq \emptyset$.

We are to show $\Omega \subset C_{n} \cap Q_{n}$. As $\Omega=\cap_{i=1}^{N} F\left(S_{i}\right) \cap\left(\cap_{k=1}^{M} E P\left(F_{k}\right)\right) \neq \emptyset$. Let $p \in \Omega$ then,

$$
\begin{aligned}
\phi\left(p, u_{n}\right) & =\phi\left(p, \Theta_{n}^{M} y_{n}\right) \\
& \leq \phi\left(p, y_{n}\right) \\
& =\phi\left(p, j^{-1}\left(\alpha_{n} j x_{n}+\left(1-\alpha_{n}\right) j A_{N}^{\lambda_{n}} x_{n}\right)\right) \\
& \left.=\|p\|^{2}-2\left\langle p, \alpha_{n} j x_{n}+\left(1-\alpha_{n}\right) j A_{N}^{\lambda_{n}} x_{n}\right\rangle+\| \alpha_{n} j x_{n}+\left(1-\alpha_{n}\right) j A_{N}^{\lambda_{n}} x_{n}\right) \|^{2} \\
& \leq\|p\|^{2}-2 \alpha_{n}\left\langle p, j x_{n}\right\rangle-2\left(1-\alpha_{n}\right)\left\langle p, j A_{N}^{\lambda_{n}} x_{n}\right\rangle+\alpha_{n}\left\|x_{n}\right\|^{2}+\left(1-\alpha_{n}\right)\left\|A_{N}^{\lambda_{n}} x_{n}\right\|^{2} \\
& =\alpha_{n} \phi\left(p, x_{n}\right)+\left(1-\alpha_{n}\right) \phi\left(p, A_{N}^{\lambda_{n}} x_{n}\right) \\
& \leq \phi\left(p, x_{n}\right) .
\end{aligned}
$$

Therefore, $p \in C_{n}$ and this implies that $\Omega \subset C_{n}$ for all $\mathrm{n} \in \mathbb{N}$. Next, we show by induction that, $\Omega \subset C_{n} \cap Q_{n}$.

From $Q_{1}=\mathrm{C}$ we have $\Omega \subset C_{1} \cap Q_{1}$. Suppose that, $\Omega \subset C_{k} \cap Q_{k}$ for some $k \in \mathbb{N}$ then, there exists $x_{k+1} \in Q_{k} \cap C_{k}$ such that,

$$
x_{k+1}=\Pi_{Q_{k} \cap C_{k}} x_{1}
$$

But, from the definition of $x_{k+1}$ we have

$$
\left\langle x_{k+1}-z, j x_{1}-j x_{k+1}\right\rangle \geq 0, \forall z \in Q_{k} \cap C_{k} .
$$


Since, $\Omega \subset Q_{k} \cap C_{k}$ then, $\forall z \in \Omega$ we have

$$
\left\langle x_{k+1}-z, j x_{1}-j x_{k+1}\right\rangle \geq 0,
$$

which implies that, $z \in Q_{k+1}$ from the definition of $Q_{n}$ in the algorithm given above. So, we have

$$
\Omega \subset Q_{k+1},
$$

for $\Omega \subset C_{n} \forall n \in \mathbb{N}$ we write

$$
\Omega \subset Q_{k+1} \cap C_{k+1},
$$

which means that, $\Omega \subset Q_{n} \cap C_{n}$ for all $n \in \mathbb{N}$. Hence, $\left\{x_{n}\right\}$ is well defined.

From the definition of $Q_{n}$, we have $x_{n}=\Pi_{Q_{n}} x_{1}$. Using lemma 2.1 we have

$$
\begin{aligned}
\phi\left(x_{n}, x_{1}\right) & =\phi\left(\Pi_{Q_{n}} x_{1}, x_{1}\right) \\
& \leq \phi\left(p, x_{1}\right)-\phi\left(p, \Pi_{Q_{n}} x_{1}\right) \\
& \leq \phi\left(p, x_{1}\right), \quad \forall p \in \Omega \subset Q_{n} .
\end{aligned}
$$

Thus, $\left\{\phi\left(x_{n}, x_{1}\right)\right\}$ is bounded. Therefore, $\left\{x_{n}\right\}$ and $\left\{S_{i} x_{n}\right\}$ are bounded for all $1 \leq i \leq N$. Since $x_{n+1}=\Pi_{Q_{n} \cap C_{n}} x_{1} \in Q_{n} \cap C_{n}$ and $x_{n}=\Pi_{Q_{n}} x_{1}$ from the definition of $\Pi_{Q_{n}}$ we have

$$
\phi\left(x_{n}, x_{1}\right) \leq \phi\left(x_{n+1}, x_{1}\right), \quad \forall n \in \mathbb{N} .
$$

Thus, $\left\{\phi\left(x_{n}, x_{1}\right)\right\}$ is nondecreasing. Therefore, its limit exists. From $x_{n}=\Pi_{Q_{n}} x_{1}$ and lemma 2.1 we also have

$$
\begin{aligned}
\phi\left(x_{n+1}, x_{n}\right) & =\phi\left(x_{n+1}, \Pi_{Q_{n}} x_{1}\right) \\
& \leq \phi\left(x_{n+1}, x_{1}\right)-\phi\left(\Pi_{Q_{n}} x_{1}, x_{1}\right) \\
& =\phi\left(x_{n+1}, x_{1}\right)-\phi\left(x_{n}, x_{1}\right), \quad \forall n \in \mathbb{N} .
\end{aligned}
$$

This means that, $\lim _{n \rightarrow \infty} \phi\left(x_{n+1}, x_{n}\right)=0$. From $x_{n+1}=\Pi_{Q_{n} \cap C_{n}} x_{1} \in C_{n}$ we have

$$
\phi\left(x_{n+1}, u_{n}\right) \leq \phi\left(x_{n+1}, x_{n}\right), \quad \forall n \in \mathbb{N} .
$$

Therefore,

$$
\lim _{n \rightarrow \infty} \phi\left(x_{n+1}, u_{n}\right)=0 .
$$

Since $\lim _{n \rightarrow \infty} \phi\left(x_{n+1}, u_{n}\right)=\lim _{n \rightarrow \infty} \phi\left(x_{n+1}, x_{n}\right)=0$, with $E$ uniformly convex and smooth, from lemma 2.2 we have

$$
\lim _{n \rightarrow \infty}\left\|x_{n+1}-x_{n}\right\|=\lim _{n \rightarrow \infty}\left\|x_{n+1}-u_{n}\right\|=0 .
$$

So, it follows that

$$
\lim _{n \rightarrow \infty}\left\|x_{n}-u_{n}\right\|=0 .
$$

Since $J$ is norm-to-norm uniformly continuous on bounded sets and $\lim _{n \rightarrow \infty}\left\|x_{n}-u_{n}\right\|=0$, we have

$$
\lim _{n \rightarrow \infty}\left\|j x_{n}-j u_{n}\right\|=0 .
$$

Let $r=\sup _{n \in \mathbb{N}}\left\{\left\|x_{n}\right\|,\left\|S_{i} x_{n}\right\|\right\}$ for $1 \leq i \leq N$. Since $E$ is a uniformly smooth Banach space, we know that, $E^{*}$ is a uniformly convex Banach space. Therefore, from lemma 2.3, there exists a continuous, strictly increasing and convex function $g$, with $g(0)=0$ such that,

$$
\left\|\alpha x^{*}+(1-\alpha) y^{*}\right\|^{2} \leq \alpha\left\|x^{*}\right\|^{2}+(1-\alpha)\left\|y^{*}\right\|^{2}-\alpha(1-\alpha) g\left(\left\|x^{*}-y^{*}\right\|\right),
$$


for $x^{*}, y^{*} \in B_{r}^{*}$ and $\alpha \in[0,1]$. So, for $p \in \Omega$ we have

$$
\begin{aligned}
\phi\left(p, u_{n}\right)= & \phi\left(p, \Theta_{n}^{M} y_{n}\right) \\
\leq & \phi\left(p, y_{n}\right) \\
= & \phi\left(p, j^{-1}\left(\alpha_{n} j x_{n}+\left(1-\alpha_{n}\right) j A_{N}^{\lambda_{n}} x_{n}\right)\right. \\
= & \|p\|^{2}-2\left\langle p, \alpha_{n} j x_{n}+\left(1-\alpha_{n}\right) j A_{N}^{\lambda_{n}} x_{n}\right\rangle+\left\|\alpha_{n} j x_{n}+\left(1-\alpha_{n}\right) j A_{N}^{\lambda_{n}} x_{n}\right\|^{2} \\
\leq & \|p\|^{2}-2 \alpha_{n}\left\langle p, j x_{n}\right\rangle-2\left(1-\alpha_{n}\right)\left\langle p, j A_{N}^{\lambda_{n}} x_{n}\right\rangle+\alpha_{n}\left\|x_{n}\right\|^{2}+\left(1-\alpha_{n}\right)\left\|A_{N}^{\lambda_{n}} x_{n}\right\|^{2} \\
& -\alpha_{n}\left(1-\alpha_{n}\right) g\left(\left\|j x_{n}-j A_{N}^{\lambda_{n}} x_{n}\right\|\right) \\
= & \alpha_{n} \phi\left(p, x_{n}\right)+\left(1-\alpha_{n}\right) \phi\left(p, A_{N}^{\lambda_{n}} x_{n}\right)-\alpha_{n}\left(1-\alpha_{n}\right) g\left(\left\|j x_{n}-j A_{N}^{\lambda_{n}} x_{n}\right\|\right) \\
\leq & \phi\left(p, x_{n}\right)-\alpha_{n}\left(1-\alpha_{n}\right) g\left(\left\|j x_{n}-j A_{N}^{\lambda_{n}} x_{n}\right\|\right) .
\end{aligned}
$$

Therefore, we have

$$
\alpha_{n}\left(1-\alpha_{n}\right) g\left(\left\|j x_{n}-j A_{N}^{\lambda_{n}} x_{n}\right\|\right) \leq \phi\left(p, x_{n}\right)-\phi\left(p, u_{n}\right), \quad \forall n \in \mathbb{N} .
$$

Since

$$
\begin{aligned}
0 & \leq \phi\left(p, x_{n}\right)-\phi\left(p, u_{n}\right) \\
& =\left\|x_{n}\right\|^{2}-2\left\langle p, j x_{n}-j u_{n}\right\rangle-\left\|u_{n}\right\|^{2} \\
& \leq\left|\left\|x_{n}\right\|^{2}-\left\|u_{n}\right\|^{2}\right|+2\left|\left\langle p, j x_{n}-j u_{n}\right\rangle\right| \\
& \leq\left|\left\|x_{n}\right\|-\left\|u_{n}\right\|\right|\left(\left\|x_{n}\right\|+\left\|u_{n}\right\|\right)+2\|p\|\left\|j x_{n}-j u_{n}\right\| \\
& \leq\left\|x_{n}-u_{n}\right\|\left(\left\|x_{n}\right\|+\left\|u_{n}\right\|\right)+2\|p\|\left\|j x_{n}-j u_{n}\right\|,
\end{aligned}
$$

for $\left\{x_{n}\right\},\left\{u_{n}\right\}$ being bounded and $\lim _{n \rightarrow \infty}\left\|j x_{n}-j u_{n}\right\|=\lim _{n \rightarrow \infty}\left\|x_{n}-u_{n}\right\|=0$, we have

$$
\lim _{n \rightarrow \infty} \phi\left(p, x_{n}\right)-\phi\left(p, u_{n}\right)=0 .
$$

From $\liminf _{n \rightarrow \infty} \alpha_{n}\left(1-\alpha_{n}\right)>0$, we have

$$
\lim _{n \rightarrow \infty} g\left(\left\|j x_{n}-j A_{N}^{\lambda_{n}} x_{n}\right\|\right)=0 .
$$

From the property of $g$, we have

$$
\lim _{n \rightarrow \infty}\left\|j x_{n}-j A_{N}^{\lambda_{n}} x_{n}\right\|=0 .
$$

As $J^{-1}$ is uniformly norm-to-norm continuous on bounded sets, we have

$$
\lim _{n \rightarrow \infty}\left\|x_{n}-A_{N}^{\lambda_{n}} x_{n}\right\|=0 .
$$

Since $\left\{x_{n}\right\}$ is bounded then, there exists a subsequence $\left\{x_{n j}\right\}$ of $\left\{x_{n}\right\}$ such that $x_{n j} \rightarrow \hat{x}$ and together with the fact of $A_{N}^{\lambda_{n}} \in V$ we have $\hat{x} \in F\left(A_{N}^{\lambda_{n}}\right) \forall N \in \mathbb{N}$. But, $F\left(A_{N}^{\lambda_{n}}\right)=\cap_{i=1}^{N} F\left(S_{i}\right)$.

Next we show $\hat{x} \in E P\left(f_{k}\right) \forall k \in\{1,2, \ldots M\}$. Now, from $u_{n}=\Theta_{n}^{M} y_{n}$, lemma 2.5 and $\phi\left(p, y_{n}\right) \leq \phi\left(p, x_{n}\right)$ we have

$$
\begin{aligned}
\phi\left(u_{n}, y_{n}\right) & =\phi\left(\Theta_{n}^{M} y_{n}, y_{n}\right) \\
& \leq \phi\left(p, y_{n}\right)-\phi\left(p, \Theta_{n}^{M} y_{n}\right) \\
& \leq \phi\left(p, x_{n}\right)-\phi\left(p, \Theta_{n}^{M} y_{n}\right) \\
& =\phi\left(p, x_{n}\right)-\phi\left(p, u_{n}\right) .
\end{aligned}
$$

So, we have

$$
\lim _{n \rightarrow \infty} \phi\left(u_{n}, y_{n}\right)=0 .
$$

Since $E$ is uniformly convex and smooth, we have from lemma 2.2 that

$$
\lim _{n \rightarrow \infty}\left\|u_{n}-y_{n}\right\|=0 .
$$


Using $\left\|x_{n}-u_{n}\right\| \rightarrow 0$ and (9) we have $y_{n k} \rightarrow \hat{x}$ and $u_{n k} \rightarrow \hat{x}$. For $J$ norm-to-norm uniformly continuous on bounded sets and (9) we have

$$
\lim _{n \rightarrow \infty}\left\|j u_{n}-j y_{n}\right\|=0
$$

From $r_{k, n} \in(0, \infty) \forall k \in\{1,2, \cdots M\}$ with $n, M \in \mathbb{N}$ we have

$$
\lim _{n \rightarrow \infty} \frac{\left\|j u_{n}-j y_{n}\right\|}{r_{M, n}}=0 .
$$

From $u_{n}=\Theta_{n}^{M} y_{n}$, we have

$$
f_{M}\left(u_{n}, y\right)+\frac{1}{r_{M, n}}\left\langle y-u_{n}, j u_{n}-j y_{n}\right\rangle \geq 0, \quad \forall y \in C,
$$

from which we have

$$
f_{M}\left(u_{n j}, y\right)+\frac{1}{r_{M, n j}}\left\langle y-u_{n j}, j u_{n j}-j y_{n j}\right\rangle \geq 0
$$

and from (A2) we have

$$
\begin{aligned}
\frac{1}{r_{M, n j}}\left\langle y-u_{n j}, j u_{n j}-j y_{n j}\right\rangle & \geq-f_{M}\left(u_{n j}, y\right) \\
& \geq f_{M}\left(y, u_{n j}\right) .
\end{aligned}
$$

Letting $j \rightarrow \infty$, we have from (10) and (A4) that

$$
f_{M}(y, \hat{x}) \leq 0, \quad \forall y \in C .
$$

Since, $f_{k}(x, y) \leq f_{k+1}(x, y) \forall x, y \in E$ then

$$
f_{k}(y, \hat{x}) \leq 0, \quad \forall y \in C \text { and } k \in\{1,2, \ldots M\} .
$$

For $0<t \leq 1$ and $y \in C$, let $y_{t}=t y+(1-t) \hat{x}$. Since $y \in C$ and $\hat{x} \in C$ we have $y_{t} \in C$ and $f_{k}\left(y_{t}, \hat{x}\right) \leq 0 \quad \forall k \in\{1,2, \ldots M\}$. So, from (A1) and (A4) we have

$$
\begin{aligned}
0 & =f_{k}\left(y_{t}, y_{t}\right) \\
& \leq t f_{k}\left(y_{t}, y\right)+(1-t) f_{k}\left(y_{t}, \hat{x}\right) \\
& \leq t f_{k}\left(y_{t}, y\right), \quad \forall k \in\{1,2, \ldots M\} .
\end{aligned}
$$

Dividing by $t$ and letting $t \rightarrow 0$, together with (A3) we have

$$
f_{k}(\hat{x}, y) \geq 0, \quad \forall y \in C \text { and } \forall k \in\{1,2, \ldots M\} .
$$

Therefore, $\hat{x} \in E P\left(f_{k}\right) \quad \forall k \in\{1,2, \ldots M\}$, which implies that $\hat{x} \in \cap_{k=1}^{M} E P\left(f_{k}\right)$. Hence, $\hat{x} \in \Omega$.

Let $h=\Pi_{\Omega} x_{1}$ from $x_{n+1}=\Pi_{C_{n} \cap Q_{n}} x_{1}$ and $h \in \Omega \subset C_{n} \cap Q_{n}$, we have

$$
\phi\left(x_{n+1}, x_{1}\right) \leq \phi\left(h, x_{1}\right) .
$$

Since a norm is weakly lower semi-continuous, we have

$$
\begin{aligned}
\phi\left(\hat{x}, x_{1}\right) & =\|\hat{x}\|^{2}-2\left\langle\hat{x}, j x_{1}\right\rangle+\left\|x_{1}\right\|^{2} \\
& \leq \liminf _{j \rightarrow \infty}\left(\left\|x_{n j}\right\|^{2}-2\left\langle x_{n j}, j x_{1}\right\rangle+\left\|x_{1}\right\|^{2}\right) \\
& =\liminf _{j \rightarrow \infty} \phi\left(x_{n j}, x_{1}\right) \\
& \leq \limsup _{j \rightarrow \infty} \phi\left(x_{n j}, x_{1}\right) \\
& \leq \phi\left(h, x_{1}\right) .
\end{aligned}
$$


From the definition of $\Pi_{\Omega}$, we have $\hat{x}=h$. Hence, $\lim _{j \rightarrow \infty} \phi\left(x_{n j}, x_{1}\right)=\phi\left(h, x_{1}\right)$ therefore,

$$
\begin{aligned}
0 & =\lim _{j \rightarrow \infty} \phi\left(x_{n j}, x_{1}\right)-\phi\left(h, x_{1}\right) \\
& =\lim _{j \rightarrow \infty}\left(\left\|x_{n j}\right\|^{2}-\|h\|^{2}-2\left\langle x_{n j}-h, J x_{1}\right\rangle\right) \\
& =\lim _{j \rightarrow \infty}\left(\left\|x_{n j}\right\|^{2}-\|h\|^{2}\right) .
\end{aligned}
$$

Since $E$ has the Kadec-Klee property, we have that $x_{n j} \rightarrow h=\Pi_{\Omega} x_{1}$, hence $\left\{x_{n}\right\}$ converges strongly to $\Pi_{\Omega} x_{1}$.

\section{Conclusion}

Nonlinear operators are of vital importance in the study/discussion of fixed point theory. It is clear that, the result obtained in this research improves the work in [15] by using a linear combination of relatively nonexpansive mappings compared to the single relatively nonexpansive map used in [15]. Also, establishing a result with different classes of nonlinear operators rather than only one class is an achievement; as the application of the result can now have wider coverage. Although, the research conducted adopt almost all the procedures and technique used in [21] with some little adjustments where necessary but, the mappings used in [21] were substituted with relatively nonexpansive mappings due to their application importance especially in resolvent of a maximal monotone operators as can be seen in [22] and therein. Future research can be conducted by using a linear combination of both the relatively nonexpansive and $\phi$-nonexpansive mappings in a single algorithm or just using mappings of more application importance.

Acknowledgments. The Author will like to thank Assoc. Prof. Bashir Ali(Phd) of the Department of Mathematical Sciences, Bayero University Kano, Nigeria for his help and immense support. Also, the author is grateful to the reviewers for their valuable suggestions that improves the quality of the work.

\section{References}

1. C.E. Chidume, Applicable Functional Analysis, I.C.T.P publicaion section, Triesty(Italy), 2003.

2. C. Zălinescu, Convex Analysis in General Vector Spaces, World Scientific River Edge, NJ, U.S.A, 2002.

3. C. Zălinescu On Uniformly Convex Functions, J. Math. Anal. Appl. 95(1983) 344-374.

4. E. Blum, W. Oettli, from Optimization and Variational Inequalities to equilibrium Problems, Math. Stud., 63(1994) 123-145.

5. G.L. Acedo, H.K. Xu, Iterative Methods for Srict Pseudo-Contractions in Hilbert Spaces, Nonlinear Anal., $67(2007) 2258-2271$.

6. H.K. Xu, Inequalities in Banach Spaces with Applications, Nonlinear Anal. 16(1991) 1127-1138.

7. I. Cioranescu, Geometry of Banach Spaces, Duality Mappings and Nonlinear Problems, Kluwer, Dordrecht, 1990.

8. P.C. Duan, J.Zhao, Strong Convergence Theorems by Hybrid Methods for Strict Pseudo-Contractions and Equilibrium Problems, FPTA, (2010), doi: 10.1155/2010/528307.

9. P. Duan, Convergence Theorems Concerning Hybrid Methods for Strict Pseudo-Contractions and Systems of Equilibrium Problems, J. Ineq. Appl.(2010), doi: 1155/2010/396080. 2010

10. P.L. Combettes, S.A. Hirstoaga, Equilibrium Programming in Hilbert Spaces, J. Nonlinear Convex Anal., 6(2005)117-136.

11. S. Kamimura, W. Takahashi, Strong Convergence of a Proximal-Type Algorithm in a Banach Space, SIAM J. Optim. 13(2002) 938-945.

12. S. S. Chang, Jong Kyu Kim and Xiong Rui Wang. Modified Block Iterative Algorithm for Solving Convex Feasibility Problems in Banach Spaces.J. Ineq. Appl., Vol.2010, Article ID 869684, 14 pages, doi:101155/2010/869684.

13. S.S. Zhang.Existence and Approximation of Solutions of Set-Valued Variational Inclusions in Banach Spaces. Appl. Math. Engl. Ed, 30(9), 1105-1112(2009), doi:10.1007/s10483-009-0904-6.

14. V. Colao, G. Marino, H.K. Xu, An Iterative Method for Finding Common Solutions of Equilibrium and Fixed Point Problems, J. Math. Anal. Appl. 344(2008) 340-352.

15. W. Takahashi, K. Zembayashi, Strong and Weak Convergence Theorems for Equilibrium Problems and Relatively Nonexpansive Mappings in Banach Spaces, Nonlinear Analysis(2008), doi: 10.1016/J.na.2007.11.031. 